\title{
SOME NOVEL INFRARED LASER DYES OF SUPERIOR PHOTOSTABILITY TUNABLE FROM 1.30 TO $1.52 \mu \mathrm{m}$
}

\author{
WEIMEI LUO, ZHENGHUA ZHU and YUGUANG YAO \\ East China University of Chemical Technology, Shanghai, People's \\ Republic of China \\ LIN YE and XIANGCHUN YUAN \\ Shanghai Institute of Optics and Fine Mechanics, Academia Sinica, \\ Shanghai, People's Republic of China
}

(Received 11 October, 1989)

\begin{abstract}
Three new heptamethine pyrylium dyes containing a disubstituted amino group on the meso position in their bridged chain were investigated. The solutions of the dyes in 1,2-dichloroethane (DCE) of concentration ca. $10^{-3} \mathrm{M}$ act as laser dyes transversely pumped by a Q-switched $\mathrm{Nd}$ :YAG laser as a pumping source. 5.6 to $9.6 \%$ of the pump energy was converted to an intense infrared pulse with frequencies between $6580 \mathrm{~cm}^{-1}(1.52 \mu \mathrm{m})$ and $7690 \mathrm{~cm}^{-1}(1.30 \mu \mathrm{m})$. The relation between lasing efficiency and dye concentration was also studied. These dyes show a remarkable photostability upon 1.06 $\mu \mathrm{m}$ laser excitation and excellent storage stability without degassing.
\end{abstract}

KEY WORDS: Infrared laser dyes, photostability, heptamethine pyrylium dyes.

\section{INTRODUCTION}

Recently, the stability of infrared-absorbing pyrylium dyes has been considerably improved by suitable rigidization of the molecular structure. Most of these dyes have an electron-withdrawing group (i.e., halogen atoms) on their meso carbon atom in the polymethine chains. ${ }^{1}$ The photostability of the dyes has been studied. ${ }^{2}$ Tunable emission between 1.2 and $1.32 \mu \mathrm{m}$ with a pulse duration of 3 ps has been demonstrated in a conventional linear cavity configuration, synchronously pumped by a mode-locked Nd:YAG laser. ${ }^{3}$ Alternative means of pumping have also been reported. ${ }^{4}$

A series of new heptamethine pyrylium dyes containing a disubstituted amino group on the meso position in the bridged polymethine chain has been prepared recently. ${ }^{5}$ This series is expected to show longer wavelength stimulated emission. With the development of optical fibers, emission around $1.30 \mu \mathrm{m}$ has prompted considerable interest in the synthesis of this kind of new dye. ${ }^{6}$

In this paper, we present data on the lasing properties of the new dyes and discuss their stability. Laser action is readily achieved in these dyes with a tuning range from 1.30 to $1.52 \mu \mathrm{m}$ under pumping with a Q-switched Nd:YAG laser. 


\section{EXPERIMENTAL}

In Figure 1, we show the molecular structures of dyes No. PY-1, PY-2 and PY-3, respectively. The dyes were dissolved in 1,2-dichloroethane (DCE) and their absorption spectra were recorded by a Shimadzu UV-365 spectrophotometer.

In our laser experiments, a Q-switched Nd:YAG laser served as pumping source. The duration of the pump pulse was $10 \mathrm{~ns}$. The pulse energy was varied between 30 and $260 \mathrm{~mJ}$ (30 mJ for measuring energy conversion efficiency and $260 \mathrm{~mJ}$ for measuring photostability).

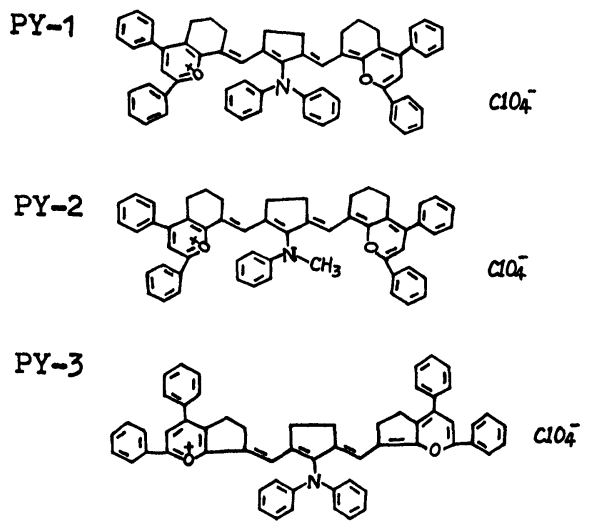

Figure 1 Structure of the investigated dyes.

The cavity for measuring energy conversion efficiency consisted of an $8 \times 8 \times 30$ $\mathrm{mm}^{3}$ quartz dye cell with one aluminum-coated side. About 1.5 milliliters of dye solution was added without stirring. The energy conversion efficiency was calculated by the following formula (1)

$$
y=E_{d} / E_{p} \times 100 \%
$$

where $y=$ energy conversion efficiency, $E_{d}=$ output energy of the tested dye laser per pulse, and $E_{p}=$ pumping energy per pulse.

The optical cavity for the tunable dye laser consisted of a diffraction grating GR ( 1200 grooves $/ \mathrm{mm}$ ) and a reflecting mirror. The infrared output was converted to the visible by using a frequency-doubling crystal BBO. The stimulated emission was analyzed by the grating monochromator SP. The energy was measured by using a joule meter. The experimental set-up is shown in Figure 2.

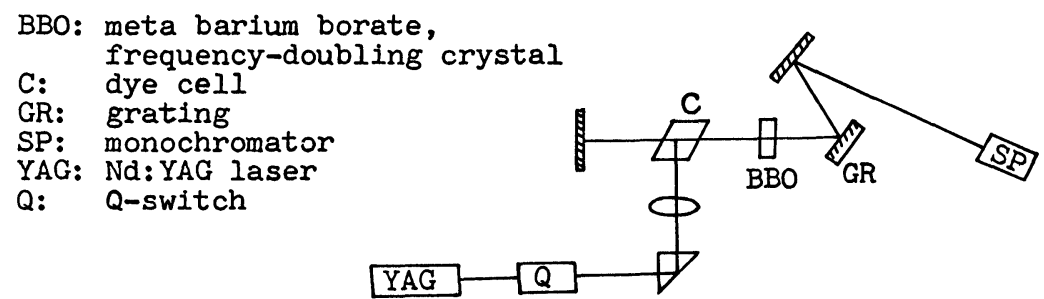

Figure 2 Schematic of the experimental set-up. 
The measurement of the photostability of the laser dyes upon $1.06 \mu \mathrm{m}$ laser excitation was carried out by observing the output energy degradation of the dye during a period of continuous vertical excitation of the 1.5 milliliters of dye solution $\left(2 \times 10^{-3} \mathrm{M}\right.$ in DCE) with a Nd:YAG laser $(260 \mathrm{~mJ}$ per pulse, repetition rate $7 \mathrm{~Hz})$.

\section{RESULTS AND DISCUSSION}

In Figure 1, the structures of these molecules are depicted. The chromophores contain symmetrical or unsymmetrical $\pi$-electron systems of the cyanine type. These molecules with long conjugated chains of extended single and double bonds were considered rather unstable; however, it has been found that they are, in fact, remarkably stable. Table 1 summarizes the photophysical properties of the dyes studied here. They show a high extinction coefficient of nearly $5 \times 10^{4}$ at $1.06 \mu \mathrm{m}$, which is important for efficient laser action. Energy conversion efficiency of the dye studied is sensitive to the concentration of the dye solution. As an example, we show the relation between dye laser output and the concentration of the dye PY-1 in Figure 3.

In Figures 4 through 6, we present the normalized absorption spectrum and the stimulated emission of the dyes. Table 2 summarizes emission parameters. The dye PY-3 shows very high lasing efficiency and a wide tuning range from 1.36 to $1.52 \mu \mathrm{m}$.

Table 1 Photophysical properties of new pyrylium dyes. Absorption maximum, $\lambda_{\max }(\mathrm{nm})$; corresponding extinction coefficient $\varepsilon\left(\lambda_{\max }\right)$; and ratio of extinction coefficients $\varepsilon(1.06 \mu \mathrm{m})$ and $\varepsilon\left(\lambda_{\max }\right)$

\begin{tabular}{lccc}
\hline Property/dye & $P Y-1$ & $P Y-2$ & $P Y-3$ \\
\hline$\lambda_{\max }$ & 1,145 & 1,150 & 1,160 \\
$\varepsilon\left(\lambda_{\max }\right) \times 10^{-3}$ & 108 & 140 & 126 \\
$\varepsilon(1.06 \mu \mathrm{m}) / \varepsilon\left(\lambda_{\max }\right)$ & 0.57 & 0.60 & 0.48 \\
\hline
\end{tabular}

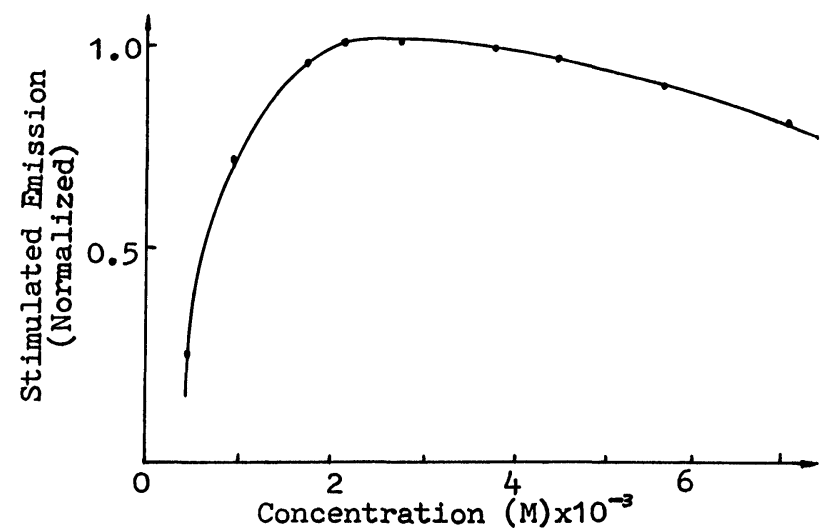

Figure 3 Relation between the relative dye laser output and the concentration of PY-1 dissolved in DCE. 


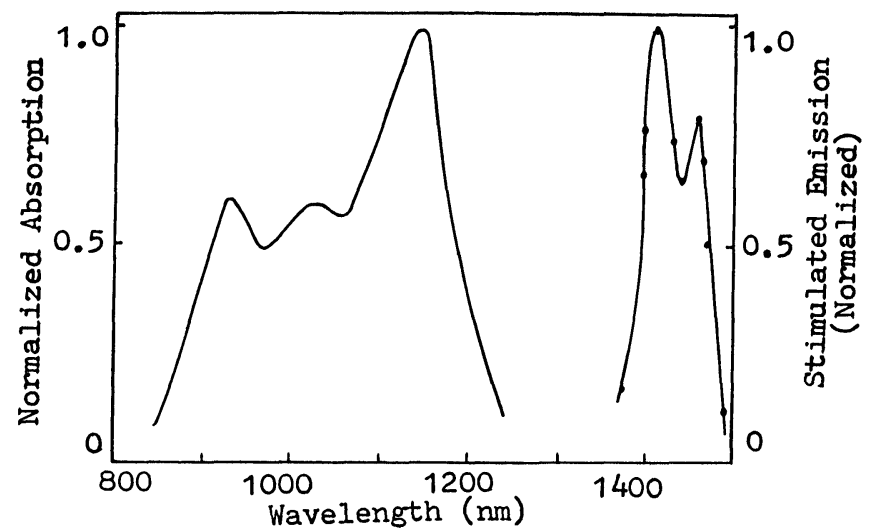

Figure 4 Normalized spectra of absorption and stimulated emission of PY-1 in DCE.

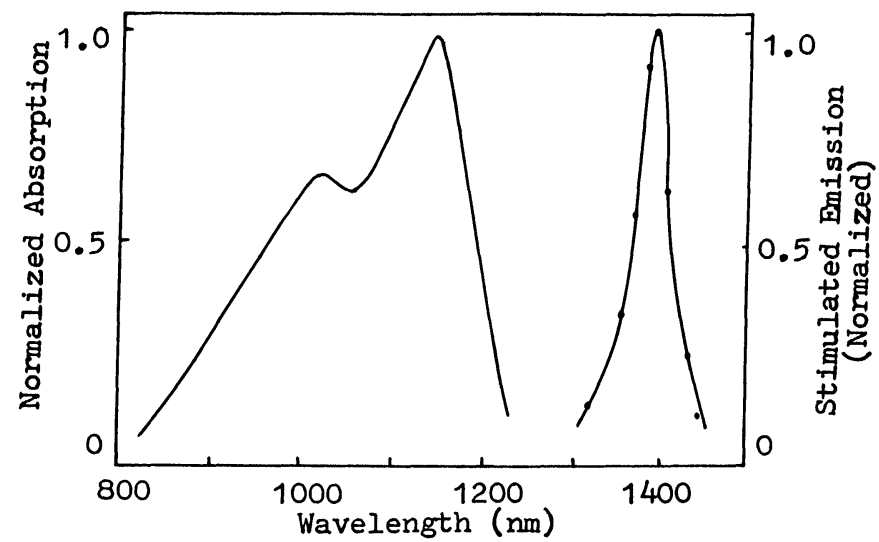

Figure 5 Normalized spectra of absorption and stimulated emission of PY-2 in DCE.

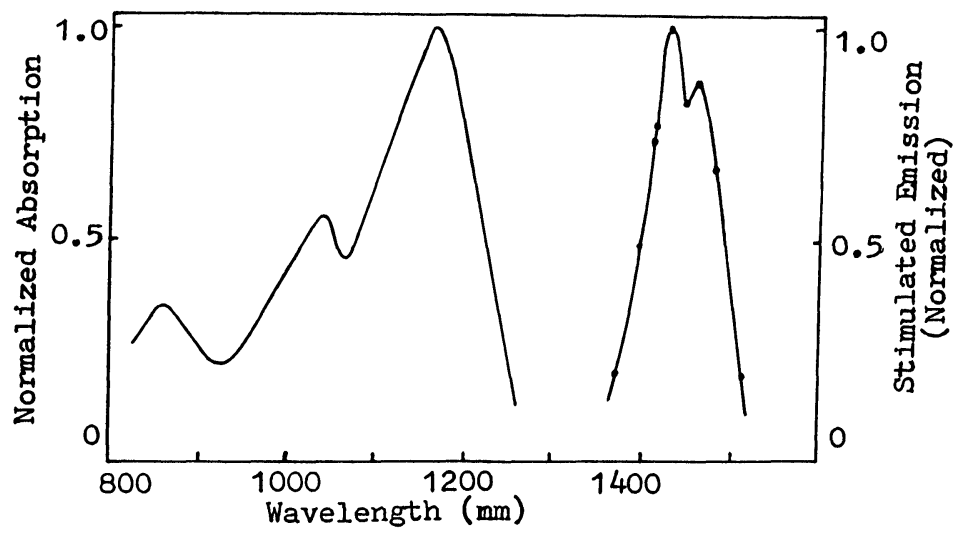

Figure 6 Normalized spectra of absorption and stimulated emission of PY-3 in DCE. 
Table 2 Parameters of stimulated emission of new pyrylium dyes in DCE: pump energy, $E_{\mathrm{p}}$; maximum of stimulated emission, $\lambda_{\mathrm{e}}$, energy conversion efficiency, $y$; pump energy threshold, $E_{\text {thr }}$; concentration, $C$; and tuning range, $R$

\begin{tabular}{lccc}
\hline Property/dye & $P Y-1$ & $P Y-2$ & $P Y-3$ \\
\hline$E_{p}(\mathrm{~mJ})$ & 30 & 30 & 30 \\
$\lambda_{e}(\mathrm{~nm})$ & 1,420 & 1,390 & 1,425 \\
$y$ & 8.2 & 7.2 & 9.6 \\
$E_{\mathrm{thr}}(\mathrm{mJ})$ & 20 & 20 & 20 \\
$C\left(10^{-3} \mathrm{M}\right)$ & 2 & 3 & 2 \\
$R(\mu \mathrm{m})$ & $1.37-1.49$ & $1.30-1.46$ & $1.36-1.52$ \\
\hline
\end{tabular}

Dye solutions in DCE with a concentration of $2 \times 10^{-3} \mathrm{M}$ show high stability when subjected to Nd:YAG laser irradiation. The energy conversion efficiency of the dyes does not decrease at all, even after being subjected to $8 \times 10^{4}$ laser pulses. Furthermore, the solutions of the dyes also show excellent storage stability. The energy conversion efficiency is not degraded at all, even after the dyes have been stored in the laboratory at room temperature for 50 days in daylight.

The high stability and remarkable lasing properties make these dyes attractive for practical applications.

\section{CONCLUSION}

Novel heptamethine pyrylium dyes with a disubstituted amino group on the meso position in their bridged chain are found to have good lasing characteristics. Pulsed laser operation with a tunability range from 1.30 to $1.52 \mu \mathrm{m}$ have been examined. Their very high photostability and storage stability make these dyes especially attractive.

\section{References}

1. G. A. Regnold and K. H. Drexhage, J. Org. Chem. 42, 759 (1977).

2. B. Kopainsky, P. Qiu and W. Kaiser, Appl. Phys. 15, 1329 (1982).

3. W. Kranitzky, B. Kopainsky and W. Kaiser, Opt. Commun. 36, 149 (1982).

4. H. Roskos, IEEE J. Quant. Electron, QE-22, 697 (1986).

5. Weimei Luo, Zhenghua Zhu and Zuguang Yao, to be published.

6. D. N. Bloom, L. F. Mollenauer, G. H. Lin, D. W. Taylor and A. M. Delgandio, Opt. Letters 4, 297 (1979). 